\title{
The Kind of Knowledge Assessed through Mature Age Entry Admission Tests in Namibia Institutions of Higher Learning: Case Study of the University of Namibia and the Polytechnic of Namibia
}

\author{
Lydia Shaketange $^{1} \&$ Alex Kanyimba $^{1}$ \\ ${ }^{1}$ Faculty of Education, University of Namibia \\ Correspondence: Alex Kanyimba, Faculty of Education, University of Namibia, Windhoek Namibia. Tel: 264- \\ 61-206-3886 \\ Received: August 15, 2016 \\ Accepted: September 15, 2016 \\ Online Published: September 20, 2016 \\ doi:10.5430/ijhe.v5n4p63 \\ URL: http://dx.doi.org/10.5430/ijhe.v5n4p63
}

\begin{abstract}
The $21^{\text {st }}$ century has witnessed the emergence of a framework for Recognition of Prior Learning (RPL) as a supplementary assessment strategy. The aim of this study is to report on the research that explored the knowledge assessed through Mature Age Entry (MAE) admission tests in Namibian institutions of higher learning. The transformative model was used to discuss the knowledge that was being assessed through these tests. The data were collected through interviews with the MAE examiners and through documentation of MAE papers. The findings suggest that despite work experience being the main requirement to qualify for MAE admission tests, the knowledge assessed during the tests is purely academic, conforming to Mode 1 knowledge. The study also found that the examiners of MAE admission tests did not understand the practice of the RPL. This scenario may have influenced assessment tools used during these tests. There is a need to distinguish between prior certificated learning and prior non-certificated learning in the MAE assessments. Therefore, the assessors at Namibian institutions of higher learning need adhere to RPL policy by accommodating certificated and prior non-certificated learning in MAE assessments.
\end{abstract}

Keywords: Recognition of prior learning, Experiential learning, Subject-based knowledge, Mature age entry, National qualification framework level descriptors

\section{Introduction}

Internationally, the $21^{\text {st }}$ century has witnessed the emergence of a framework for Recognition of Prior Learning (RPL). This framework is perceived as an alternative strategy of assessment in which previously uncertificated learning is assessed to determine the extent to which such learning has achieved the required outcomes or standards for entry into a program or completion of a qualification (Harris, 2000). Learning is a complex domain, with various forms. Formal learning obtained from courses and training accredited by accrediting institutions and non-formal learning are acquired from courses and trainings that are not accredited. Unless learning is specified, it becomes impossible to differentiate between what is and what is not accredited.

The practice of RPL by various countries resulted in the development of several acronyms referring to the same concept. For example, in the United Kingdom (UK), RPL is referred to as Accreditation of Prior Learning (APL), while in the United States of America (USA) the term is construed as Prior Learning Assessment (PLA). In Australia and South Africa the acronym RPL is used for Recognition of Prior Learning (Harris, 2006). The Namibia Qualifications Authority (NQA) uses the concept RPL to define the official acknowledgement of previously acquired learning. This definition does not specify whether or not the type of learning referred to is accredited. The Namibian national policy on RPL defines the term by drawing a distinction between certificated and non-certificated learning. Thus, the term is defined as the recognition of learning attainments previously certificated and abilities that were not previous certificated. In this study, RPL is employed synonymously with the term experiential learning. In the understanding of this study, experiential learning is referred to as non-certificated learning derived from a variety of sources, including work-based training, professional conferences, on the job and through community involvement. The notion of experiential learning follows international trends which maintain that credit is never awarded as a result of experience alone, but as a result of the reflection of what is acquired from experience (Michelson et al., 2004). 
The establishment of the RPL concept in Namibia is linked to the country's historical perspectives on colonization and the apartheid system. The latter reinforced the principles of Bantu education and encouraged school segregation and imbalances in the allocation of resources (Namibia, Ministry of Education and Culture, 1993). These imbalances created a lack of interest and participation in educational activities among Namibian learners (Cohen, 1994) and resulted in the deprivation of Namibians in terms of knowledge and skills necessary for further education. However, after independence in 1990, Namibia witnessed a greater demand for social inclusion in all aspects of its economy, which necessitated the widening of access to higher education to reinforce the global trend of work and learn. The demand armored the notion of collaboration between higher education and the world of work. This implies that employees can take up study opportunities at institutions of higher learning to upgrade their knowledge, and eventually return to their workplaces with improved knowledge and qualifications, leading to improved production and income to sustain quality of life (Namibia, Ministry of Education and Culture, 1993).

The University of Namibia (UNAM) and the Namibia University of Science and Technology (NUST, formerly known as the Polytechnic of Namibia (PoN - the term used in this study), have a system in place called Mature Age Entry (MAE). This system admits adults who do not possess specified minimal entry requirements to higher education, but who have extensive work experience. The process commences with the advertisement of the service in local newspapers for eligible candidates to apply. The conditions of eligibility include a Grade 10 certificate accompanied by three to five years' relevant working experience in the field of interest. Successful candidates are invited to write an admission test. The test question papers and corresponding marking guides are prepared by examiners (usually lecturers who teach the specific subject).

In view of this, this study reports on the kind of knowledge assessed through MAE admission tests in Namibian institutions of higher learning. It postulates that examiners' understanding of the concept 'experiential learning' influences the assessment tools used during the MAE admission tests. This study therefore follows the sequence below:

1. Presentation of the problem statement;

2. Discussions of the conceptual and theoretical perspectives;

3. Exposition of the research design, strategy and methodology;

4. Presentation and interpretation of data;

5. Discussions in view of the theoretical framework;

6. Recommendations to address constraints in accessing Namibian institutions of higher learning.

\section{Statement of the Problem}

The assessment of RPL or experiential learning is internationally justified and valued through policies and frameworks (Joosten-ten et al., 2009). In Namibia, several calls have been made through various government policies (NDP3, 2001 to 2011, Vision 2030, 2004; National Policy on RPL, 2010) to widen access through alternative paths to higher education. However, no specifications have been put forward as to the forms of knowledge to be assessed through these alternative paths. Stendlund (2010) states that specifications of knowledge contribute to defining the exactness of knowledge forms that create the foundation of practice. The purpose of this study was to explore the nature of knowledge assessed through MAE admission tests at UNAM and PoN. The study also explored the examiners' understanding of work experience and whether such understanding influenced the development of the assessment tools used in MAE admission tests. The following questions were used to address the research problem:

1. What kind of knowledge is assessed through MAE admission tests in Namibian institutions of higher learning?

2. How is RPL viewed and perceived by examiners of MAE admission tests in Namibian institutions of higher learning?

3. How is RPL distinguished from academic learning (Mode 1 knowledge) in Namibian institutions of higher learning?

\section{Conceptual and Theoretical Framework}

The concept of knowledge is important in the empirical part of this article because it was used to assess the MAE admission tests in Namibian institutions of higher learning. It is explained to demonstrate the context within which it is used here. The manner in which the concept becomes subsumed into the three RPL models of assessment is explained. 
Harris (2000) and Mankin (2009) categorized knowledge into modes: Mode 1 and Mode 2 knowledge. Mode 1 knowledge is linked to knowledge produced by academics through scientific and disciplinary subject content. This knowledge is about understanding of scientific facts and concepts and is encoded through books and disciplinary articles, taking the form of who knows what. People are aware of the existence of this knowledge because it is synchronized through academic books. Therefore, it is perceived as normal and straightforward (Harris, 2000; Mankin, 2009).

Mode 2 knowledge is perceived as stemming from the context of practice or a person's skills component. The characteristics of Mode 2 knowledge are heterogeneity, and diversity and social reflexivity. Thus, Mode 2 knowledge is not synchronized through a discipline but resides within the individual's skills base or areas of professional expertise. Mankin further states that this knowledge is context specific, implicit, socially produced and usually acquired from non-accredited courses. According to Mankin (2009), this form of knowledge is developed from personal experience and resides within the individual's judgment and intuition. It takes the form of who knows how. Since this knowledge is embedded within an individual's experience and judgment, it is invisible, difficult to identify and articulate across contexts (Harris, 2000; Mankin, 2009).

\subsection{The models of Assessment in RPL}

Three perspectives which can be used to portray the assessment of RPL in institutions of higher learning are reflected in the literature. These models are discussed below to demonstrate their implication and relevance to Mode 1 and Mode 2 knowledge in the assessment of RPL through the MAE Admission in Namibian institutions of higher learning.

\subsection{The Credit Exchange (Procrustean) Model}

The credit exchange (Procrustean) model advocates liberal learning and maintains that knowledge is based upon empirical investigation and governed by technical rules (Mode 1 knowledge). This means that the institution must determine and specify the competency, skills and knowledge to be assessed. In this model, the candidates must offer evidence of their learning by correctly responding to demands of the host institution. Evidence of learning is assessed by comparing candidates' learning against defined competencies skills and knowledge that the institution requires. Critics of this model have argued that this it favors candidates whose job performances indicate familiarity with the knowledge required by the host institution (Ralphs, 2008)

\subsection{The Developmental Model}

The developmental model falls under the humanistic approach and advocates freedom and autonomous learning (Suopis, 2009; Ord, 2009). This model advocates that learning should arise from social and life context and encourages candidates to write what they have learnt from work experience. The candidates are the masters of what they know and develop the content of a portfolio of evidence which contains all learning acquired. The portfolio of evidence becomes the curriculum through which their learning is assessed (Michelson, 2004). The model requires that candidates be assisted in developing and compiling their portfolios. Evidence of experiential learning is matched against the required academic knowledge. Critics of this model have censured the model's adherence to reflective exercise and argue that it does not resist the power to match the candidate's experiential learning against predefined academic knowledge of institutions (Harris, 1999).

\subsection{The Transformative (Radical) Model}

The transformative model is associated with trade unions, feminists groups and critical theory that view education as a means to transform an individual and society (Breier, 2005). These movements propose that education is a force for achieving radical social change. Grounded in the notion that all knowledge is performative, prior non-certificated learning is perceived as knowledge derived from events that workers have lived. Knowledge is perceived in its own right, within its context of practice and can be accepted even if it is not aligned to academic merit. This entails that candidates who enter higher education through RPL do so not because their competencies meet the institution's prescribed knowledge, but because the institution recognizes the existence and diversity of knowledge they possess. Harris (1999) and Volbrecht (2009) acknowledge this recognition and also assert that the transformative model is no less trapped in a modernist frame, and that its epistemological understanding of knowledge is not different from credit exchange and developmental models of RPL. Stuckey et al. (2014) encourage institutions of higher learning to engage in the ideology of transformative learning in both their teaching and assessment.

The study view is that the assessment of MAE admission tests in Namibian institutions of higher learning should be based on the transformative model. This model is consistent with Mode 2 knowledge because of its insistence on heterogeneity and diversity and social reflexivity. It resides within the individual's skills base or areas of professional 
expertise. Some examples of the application of this model in institutions of higher learning can be cited. For example, the University without Walls in the USA has assessed experiential learning and matched learning evidence to academic learning by identifying areas of competences in the candidate's field of interest (Suopis, 2009).

A candidate who applies for Management courses would for instance be required to construct six areas pertinent to Management, such as customer services, retention and many others, and write six to ten pages about learning gained through work experience on each of the six topics. This helps to extract learning from experiences that were encountered. The London Metropolitan University credits applicants who demonstrate how learning from experience by articulating a series of learning outcomes that match their experiential learning and that relate to the subject matter applied for (Michelson, 2004). Most institutions of higher learning are faced with the dilemma of aligning experiential learning to knowledge required without immense prestige of one form of knowledge over the other. Joosten-ten Brinke et al. (2009) argue that candidates should be assessed on the extent to which they have fulfilled the requirement and not necessarily the methods and content by which they have acquired the knowledge they possess.

Based on the earlier mentioned conceptual and theoretical framework, this study was undertaken to explore the knowledge that is assessed through MAE admission tests in Namibian institutions of higher learning.

\section{Methods}

A qualitative approach (Henning et al., 2004; Creswell, 2009; Neumann, 2011) was employed to solicit participants' views. A case study design (Creswell, 2009) was used to solicit cases of knowledge assessed through MAE admission tests from UNAM and PoN and to explore the examiners' understanding of assessing work experience. The qualitative approach fits well with transformative model as both are grounded in the view that reality is socially constructed (Cohen et al., 2010).

\subsection{Population and Sampling}

A purposive sampling strategy (Patton, 2002; Merriam, 2009; Coles and McGrath, 2010) was used to select respondents from the population of 2,029 lecturers at the two institutions. By virtue of their professional roles, 17 lecturers, referred to in this study as assessors, were sampled. The study is focused on accessing respondents who have experience of MAE assessments. Therefore, assessors with more than two years' experience in setting and marking MAE tests were selected, while those with more than two years' experience in assessing portfolios of evidence were sampled instead.

\subsection{Pilot study, Data Collection Process and Data Analysis}

A pilot study was conducted at the International University of Management (IUM) to test the instruments' appropriateness and clarity (Patton, 2002). Although IUM is a private university, its requirements for MAE admission tests are similar to those of UNAM and PoN. Four assessors were sampled and interviewed at IUM. As a result of the pilot study, two separate instruments were finally designed for assessors and a document review guide. Some research questions were refined and some were rephrased.

A one-to-one interview was employed in the data collection process. The assessors' interview guide solicited the knowledge that was being assessed through MAE admission tests and assessors' understanding of experiential learning. Institutional documents such as MAE test question papers of UNAM and PoN were purposively selected (Coles \& McGrath, 2010) as data sources so that the kind of knowledge assessed therein could be examined. The document review of the MAE test papers and interview guides were significant instruments because they provided data used in the comparison and triangulation.

Data were obtained from 17 participants for hand-analysis. Hand-analysis provides an opportunity for the researchers to become more familiar with the data (Creswell, 2008). This data analysis started with the transcription of interviews into text, organizing data into the groups of institutions from where they were collected and collated by units of analysis (Creswell, 2009). This means data were grouped according to institutional assessors and MAE question papers. The transcriptions were read and colored pencils used to mark related answers from the units of analysis. Categories were formed by grouping related answers and labeling them. A process of comparisons between properties in categories was followed to form broad themes (Creswell, 2009). Data were linked and aggregated across broad themes until manageable size of themes emerged. 


\section{Presentation and Interpretation of Data}

\section{Knowledge assessed through MAE admission tests at Namibian institutions of higher learning}

This section addresses the English Language Proficiency test, Numeracy, General Knowledge, and Specific Subject Matter tests

\subsection{Knowledge Assessed through English Language Proficiency Tests at Both Institutions}

The kind of knowledge assessed through English Language Proficiency tests at both institutions was assessed and the results are presented in Table 1.

Table 1. The kind of knowledge assessed through the English Language Proficiency test paper

\begin{tabular}{ll}
\hline UNAM & I assess the ability of candidates to read and answer questions correctly and with \\
Assessors & frequency, to scan and summarize the text in own words, and to interpret information in \\
a text. I assess the ability to identify main ideas from a given text and the ability to write \\
academically. I assess whether or not a candidate can manage the academic demands \\
once admitted.
\end{tabular}

Table 1 shows that assessors at UNAM test the correctness of answers, frequency of reading and essay writing. They also test the ability to scan and summarize texts. The assessors at PON test reading and writing skills, the ability to express oneself and grammatical correctness. These responses show that both institutions address pure academic language matters, which conforms to Mode 1 knowledge.

\subsection{Knowledge Assessed through the Numeracy Test at Both Institutions}

The knowledge assessed through the Numeracy test paper is presented in Table 2.

Table 2. The knowledge assessed through the Numeracy test paper

\begin{tabular}{ll}
\hline $\boldsymbol{U N A M}$ & $\begin{array}{l}\text { I assess numerical abilities to calculate distances, measurement, weight, defining } \\
\text { of concepts and the ability to think logically. I also assess aptitude areas such as } \\
\text { graphs, and statistics. }\end{array}$ \\
\hline$\underline{\boldsymbol{P o N}}$ & $\begin{array}{l}\text { We assess aptitude areas, basic numeracy and basic statistic, graphs, statistics, } \\
\text { mass, distance and weight. }\end{array}$
\end{tabular}

Table 2 indicates that assessors at both institutions assess distances, measurements, mass, weight, and knowledge of concepts, graphs and statistics. Both institutions assess subject knowledge that is compliant with Mode 1 knowledge.

\subsection{Knowledge Assessed through the UNAM General Knowledge Test Paper}

The assessor clarified what knowledge is assessed through the General Knowledge test paper as follows:

I test candidates on knowledge of the happenings as reported on national media. I look for knowledge of what is happening in Namibia and beyond.

The response above shows that this paper assesses the knowledge of both national and international happenings and that the knowledge being tested does not necessarily come from work experience.

\subsection{Knowledge Assessed through the UNAM Specific Subject Matter Test Paper}

The assessors' explanation as to what knowledge they look for in the Specific Subject Matter test paper is indicated in Table 3. 
Table 3. The kind of knowledge assessed through the UNAM Specific Subject Matter test paper

\begin{tabular}{|c|c|c|c|c|}
\hline \multicolumn{5}{|c|}{ What knowledge do you look for in Specific Subject Matter? } \\
\hline Assessor 1 & $\underline{\text { Assessor } 2}$ & $\underline{\text { Assessor } 3}$ & Assessor 4 & $\underline{\text { Assessor } 5}$ \\
\hline $\begin{array}{l}\text { I assess whether } \\
\text { the candidate can } \\
\text { do practical in } \\
\text { relation to the } \\
\text { subject content. }\end{array}$ & $\begin{array}{l}\text { I assess whether the } \\
\text { candidate has } \\
\text { background } \\
\text { knowledge of the } \\
\text { subject matter and if } \\
\text { they have the right } \\
\text { experience. }\end{array}$ & $\begin{array}{l}\text { I look for commitment } \\
\text { and for dedicated } \\
\text { people. }\end{array}$ & $\begin{array}{l}\text { I look for prior } \\
\text { knowledge } \\
\text { candidates, skills } \\
\text { and basic attitudes } \\
\text { needed in the field. }\end{array}$ & $\begin{array}{l}\text { I assess ideas of } \\
\text { professionalism and } \\
\text { ethics because the } \\
\text { field needs the } \\
\text { application of these. }\end{array}$ \\
\hline
\end{tabular}

Table 3 shows that assessors assess practical knowledge, background knowledge of the subject matter, commitment, prior knowledge, and professionalism. These responses seem to imply the lack of specific guidelines of knowledge to be assessed in the MAE test paper. It demonstrates that each assessor is free to assess any form of knowledge deemed fit at the time of assessment.

5.4 Knowledge Assessed through the PoN Portfolio of Evidence Test Paper

The knowledge assessed through PoN's Portfolio of Evidence test paper is shown in Table 4.

Table 4. The kind of knowledge assessors assess through Portfolio of Evidence test paper

\begin{tabular}{|c|c|}
\hline Assessors & Responses \\
\hline 1 & I ask students to submit qualifications that were obtained before entering PoN. \\
\hline 2 & I look for academic abilities and the ability to think critically. \\
\hline 3 & $\begin{array}{l}\text { We assess applicants'_attainment of equivalency to academic knowledge, skills of the subject } \\
\text { matter and whether corresponding notional hours were attained. }\end{array}$ \\
\hline 4 & $\begin{array}{l}\text { I assess whether the content of the portfolio has attained } 80 \% \text { of the course or module content } \\
\text { required for exemption. }\end{array}$ \\
\hline 5 & We need to see that the content of assessment resembles the evidence submitted. \\
\hline 6 & $\begin{array}{l}\text { We assess whether the student has the work experience that can cover } 80 \% \text { or more of what is } \\
\text { offered by PoN. }\end{array}$ \\
\hline 7 & $\begin{array}{l}\text { We assess work experience indirectly; the questions are set in such a way that the person with } \\
\text { work experience in my view can answer them. }\end{array}$ \\
\hline
\end{tabular}

5.5 Knowledge Assessed from both UNAM and PoN MAE English Language Proficiency Test Papers (2010- 2011)

Knowledge assessed through the PoN Portfolio of Evidence assessed from both UNAM and PoN MAE English Language Proficiency test papers 2010 to 2011 is presented in Table 5. 
Table 5. Knowledge assessed from both UNAM and PoN MAE English Language Proficiency test papers (20102011)

\begin{tabular}{llll}
\hline Year & Subject & UNAM & PoN \\
\hline 2010 & English & The English Language & The English Language Proficiency test \\
& Language & Proficiency test had three required candidates to write an essay of not \\
& Proficiency test & sections consisting of reading more than 250 words on one of five topical \\
& paper & comprehension passages issues. Identification of errors in given \\
& followed by answering of sentences, completing of sentences, reading \\
& questions from the passage. comprehension and answering to questions, \\
& Finding words from the passage arranging sentences in correct order.
\end{tabular}

2011

\section{English Language Proficiency}

test paper

2010

Numeracy

2011

\section{Portfolio of Evidence} that were synonymous with words provided. True or False questions. Grammar section and essay writing section.

The test paper consisted of two sections. Section one required candidates to read a given text, and to answer subsequent questions, choose and tick the best option for completing a sentence, giving synonymous to given words. Section two comprised essay writing (150 words required) on a given topic.

Numeracy MAE test paper required candidates to answer multiple questions. Candidates were given a list of abbreviations to find the most appropriate abbreviations,

Circle correct answer, rounding off answers to one decimal place, multiple choice questions to determining right answers, use of mass measurements and distance.
The test paper was written in 2010/2011, indicating that this same paper was written for 2010 and 2011 admission tests.

Candidates were required to write down the formula of getting correct answers to specific sums given, knowledge of inches, meters, true or false calculations, reading of pie charts, knowledge soliciting exercises such as 'a man has 32 teeth and lost three teeth, how many teeth has he lost?'.

Writing numbers in sequence, calculation of age differences as per given exercise, calculations of mass, meters, true or false statements, household finance.

The researcher was not able to access copies of portfolios of evidence which were compiled by students.

Table 5 shows that the assessment of English Language Proficiency at UNAM and PoN was similar. This was because they all assessed the ability to answer questions correctly, including comprehension questions, grammar and the ability to write essays. The data indicate that the knowledge solicited through the MAE test papers concurs with how assessors assess mathematical formula, reading charts, use of masses and measurements. In general, the data reveal no assessment of experiential learning in either the assessors' interview responses or MAE test question papers at both UNAM and PoN. All the interviewees' responses and exercises presented in the test papers indicate disciplinary knowledge, which conforms to Mode 1 knowledge.

\subsection{Examiners' Understanding of Experiential Learning in Namibian institutions of Higher Learning}

Assessors were asked to explain what Experiential Learning is and their responses are illustrated in Table 6. 
Table 6. Examiners' understanding of the concept 'Experiential Learning'

\begin{tabular}{|c|c|c|}
\hline Assessors & UNAM & PoN \\
\hline 1 & $\begin{array}{l}\text { It is an experience that proves } \\
\text { the person has been working or } \\
\text { has worked. }\end{array}$ & $\begin{array}{l}\text { Declined to define experiential learning because } \\
\text { had no idea. }\end{array}$ \\
\hline 2 & $\begin{array}{l}\text { It is learning that happens while } \\
\text { working in the field. }\end{array}$ & Declined to define because did not know. \\
\hline 3 & $\begin{array}{l}\text { It is about practicing the skills } \\
\text { informally without the academic } \\
\text { component. }\end{array}$ & $\begin{array}{l}\text { Declined to define as was unable to give the } \\
\text { definition. }\end{array}$ \\
\hline 4 & $\begin{array}{l}\text { It is having been working for } \\
\text { years such as five years. }\end{array}$ & $\begin{array}{l}\text { Decline to define as was unable to give the } \\
\text { definition. }\end{array}$ \\
\hline 5 & $\begin{array}{l}\text { It could be a course or program } \\
\text { which prepares people before } \\
\text { entering a program. }\end{array}$ & $\begin{array}{l}\text { It is a type of learning or acquiring knowledge } \\
\text { through experience not through official journals. }\end{array}$ \\
\hline 6 & $\begin{array}{l}\text { Learning about things through } \\
\text { life. }\end{array}$ & $\begin{array}{l}\text { It is the experience one has gained on the job, but } \\
\text { this learning is not certified. }\end{array}$ \\
\hline 7 & $\begin{array}{l}\text { Things we learn as we go } \\
\text { through life. This learning is not } \\
\text { certificated. It is your experience } \\
\text { in life. }\end{array}$ & $\begin{array}{l}\text { It is when a person has learnt something but } \\
\text { never received a certificate or diploma. The } \\
\text { person has knowledge but he or she cannot show } \\
\text { it. }\end{array}$ \\
\hline 8 & $\begin{array}{l}\text { It is a learning that is acquired } \\
\text { through work experiences or } \\
\text { through maturity. }\end{array}$ & $\begin{array}{l}\text { It is a non-certificated learning gained through } \\
\text { work experience not acquired through formal } \\
\text { classes. }\end{array}$ \\
\hline 9 & & $\begin{array}{l}\text { It is how to assess a person's knowledge that is } \\
\text { not gained through theoretical book knowledge. }\end{array}$ \\
\hline
\end{tabular}

Assessors at UNAM and PoN perceive Experiential Learning as happenings, experience, practicing skills, and learning from experience of a program. The participants equated RPL/experiential learning with events, some referred to it as learning acquired outside the formal system or learning acquired differently from the traditional way. The responses depicted experiential learning as learning that has no proof. It is an event being experienced in the job environment. The data do not give an understanding of what RPL is about. 
5.6 Assessment of RPL through MAE Admission Tests

Assessors indicate how they assess RPL in the admission tests in Table 7.

Table 7. How RPL is assessed in MAE test papers

\begin{tabular}{|c|c|}
\hline Assessors & UNAM \\
\hline 1. & $\begin{array}{l}\text { Yes. We assess RPL because of the } \\
\text { nature of questions asked in the test } \\
\text { papers. }\end{array}$ \\
\hline 2. & $\begin{array}{l}\text { No, because two hours taken to } \\
\text { write MAE tests is too little to } \\
\text { express what you know. Also, the } \\
\text { framework and structure in which } \\
\text { these MAE people write do not } \\
\text { conform to RPL. }\end{array}$ \\
\hline 3. & $\begin{array}{l}\text { No. We do not look at RPL or work } \\
\text { experience whatsoever. }\end{array}$ \\
\hline 4. & $\begin{array}{l}\text { Yes. If the candidate has worked in } \\
\text { the field, that is what we ask for. }\end{array}$ \\
\hline 5. & $\begin{array}{l}\text { Yes. Although we do not have } \\
\text { proper instruments that are } \\
\text { developed to really do a formal } \\
\text { form of assessment. }\end{array}$ \\
\hline 6. & $\begin{array}{l}\text { We definitely assess RPL. We } \\
\text { assess what these people taught } \\
\text { themselves in the field. }\end{array}$ \\
\hline 7. & $\begin{array}{l}\text { Yes. Most of the exercises that we } \\
\text { bring in the test papers are related } \\
\text { to experience in the field. }\end{array}$ \\
\hline
\end{tabular}

8.

Not sure.

Eight assessors at UNAM were interviewed and these assessed MAE tests while only two assessors at PoN assessed MAE tests. All the assessors assessed English and Numeracy while the rest of the PoN assessors who were sampled assessed portfolios of evidence. Table 7 shows contradictions of assessors for MAE in terms of RPL/experiential learning assessment. Some of assessors (6) agreed that they assessed RPL/experiential learning while others (3) denied having assessed RPL in MAE tests. Other assessors were not sure whether or not they assessed RPL, which reveals a lack of coherent understanding of the concept RPL among MAE test examiners. This can be problematic in the implementation and assessment of experiential learning because some MAE test examiners might not have been aware whether or not they were assessing RPL, while others may seem to have believed that they were assessing RPL while in fact they were assessing disciplinary knowledge. The fact that some assessors were not sure whether or not they assessed RPL indicates voluntarism of RPL in their assessment and implies that RPL was not institutionalized.

\section{Discussions}

\subsection{The Kind of Knowledge That the Assessors of MAE Assess in Namibian Institutions of Higher Learning}

The kind of knowledge assessed in the MAE tests in Namibian institutions of higher learning is purely academic. It is categorized as Mode 1 knowledge because it is generated within a discipline and is primarily cognitive. These can be averred because MAE tests are about giving correct answers to questions, to be acquainted with parts of speech and to be familiar with scanning and summarizing. The knowledge assessed in the MAE tests also includes parts of speech, interpreting graphs and statistics as well as measurements. It seems that the knowledge desired exclude knowledge produced in the context of application and organizational diversity.

Work experience is the pre-requisite for the MAE admission tests in Namibian institutions of higher learning (UNAM, Faculty of Education Prospectus, 2011). However, the data reveal a presence of pure academic or the 
so-called Mode 1 knowledge, as the sole knowledge assessed by NIHL through MAE admission tests. Osman (2003) argues that most administrators implementing RPL in higher education appear to be attracted to academic knowledge, since it resembles the knowledge used in higher education and lecturers can easily transfer academic norms with this knowledge. This scenario is disadvantageous to the majority of candidates who are likely to seek entry to higher education through MAE.

These are mainly older, non-traditional students, including active government employees, community activists, accountants and managers in organizations with applied and organizational diversity. Those candidates would not be able to recall areas of English language and Numeracy required by institutions. Conrad (2010) concurs with this conclusion because he argues that power, pedagogy and the traditional role of higher education tend to prevail as gatekeepers of institutions of higher learning whose duty it is to protect and defend the tradition of those institutions.

Based on the data presented earlier, the following conclusions can be made: First, it should be clarified that the knowledge assessed at UNAM through general knowledge and specific subject matter does not specify the purpose of assessment. Second, it is not clear what general knowledge candidates is required to possess and how such knowledge contributes to assessing their suitability and capability to access higher learning. It seems that there are no guidelines on what knowledge needs to be assessed in specific subject matters.

\subsection{How Assessors View and Perceive Experiential Learning in Namibian Institutions of Higher Learning}

Experiential learning is viewed and perceived by examiners of MAE admission to be a process of happenings and practicing skills learned from the programs. This examiners' understanding contradicts what is portrayed in the MAE admission test papers because exercises require candidates to recite knowledge generated within a discipline. According to Ord (2009), deliverance from lived experience does not imply reciting the events of what happened; rather it is the engagement with experience that creates learning. Ord recommends engagement of candidates with experience. The MAE tests do not indicate the engagement of candidates with their experience but indicate comparison to academic knowledge. Therefore, the exercises simply compel candidates to recite from knowledge which is generated in a discipline.

\subsection{How Assessors in Namibian Institutions of Higher Learning Distinguish RPL from Academic Learning}

It seems that the assessors of MAE, from both UNAM and PoN, do not distinguish between RPL and disciplinary knowledge. This can be averred because they agreed that they assess experiential learning while their assessment tools in fact assessed academic Mode 1 knowledge which is generated in a disciplinary context. For example, one of them commented that "most of the exercises that we bring in the test papers are related to experience in the field". This assertion and the type of questions asked seem to demonstrate that the assessors did not realize the distinction between RPL and academic learning. They seem to have concluded that giving exercises generated in disciplinary context is experiential learning. This lack of distinction could prove critical, particularly when the assessors develop assessment instruments because they assume that they are testing experiential learning when in fact they are entangled in traditional assessment methods.

The above finding is in line with Conrad (2009), who affirms that if the language that explains and describes the process of experiential learning is not clearly defined and articulated, experiential learning can be easily entangled with better known and understood concepts. Conrad's observation fits the Namibian situation in which assessors assumed they were assessing experiential learning when in a matter of fact they were not. Two explanations can fit this situation: Firstly, there was confusion in the coordination of policy and practice at the two institutions, where policies were enacted by authority concerning the assessment of experiential learning and such policies were disseminated without clear explanations regarding implementation. Secondly, the assessors neither knew nor realized the existence of experiential learning and the need for it in the actual assessment process.

\section{Recommendations and Policy Implications}

The study recommends the following:

- There is a need to distinguish between Mode 1 knowledge and Mode 2 knowledge in the assessments. Mode 2 knowledge is important because it is consistent with the transformative model for the assessment of RPL. This model recognises the diversity of knowledge among candidates and takes into account prior non-certificated learning.

- There is a need to assess experiential knowledge. This process should take place through aligning candidates' prior non-certificated learning against outcomes derived from the NQF Level descriptors rather than against academic knowledge. The alignment of outcomes from NQF Level descriptors with candidates' learning is not necessarily based on what is learnt or how it was learnt, but rather on whether what was learnt reflects the standards 
and volume of the level needed to enter a specific program in higher education. Specific subject matter does not specify the purpose of assessment.

- There is a need to develop guidelines for the assessment of non-certificated learning in specific subject matters that will show how to assess non-certificated learning in MAE examinations.

- There is a need to restructure MAE admission tests so that people's work experience is assessed in relation to the subject applied for. This can only be made possible with the inclusion of Mode 2 knowledge in MAE tests. The view of the authors is that Mode 2 knowledge provides educational experience that adds value to institutions and can promote an inclusive tendency in higher educational provision.

- The office of the registrar at both institutions should consider reviewing their policies concerning the MAE entry test and should clarify Mode 1 and Mode 2 knowledge.

- There is a need for clarification of knowledge assessed at UNAM through general knowledge and also specification purposes of assessment. The papers do not specify the knowledge that candidates are required to possess and how such knowledge contributes to assessing their suitability and capability to access higher learning.

- There is a need for Namibian institutions of higher learning to adhere to the national RPL policy by accommodating Mode 2 knowledge in the assessment of RPL. Other Namibian policies that complement the RPL Policy need to be improved to adhere to the classification of Mode 1 and Mode 2 knowledge. For example, the Namibia Qualification Authority (NQA, 2010) defines RPL as "previous learning acquired through any context". However, the NQA definition has not specified the type of learning being referred to as certificated or non-certificated/ Mode 1 and Mode 2 knowledge.

\section{Conclusion}

The study explored the kind of knowledge assessed through MAE admission tests in Namibian institutions of higher learning. The following research questions were addressed:

(1) What kind of knowledge is assessed through MAE admission tests in Namibian institutions of higher learning?

(2) How is RPL viewed and perceived by examiners of MAE admission test in Namibian institutions of higher learning?

(3) How is RPL distinguished from academic learning (Mode 1 knowledge) in Namibian institutions of higher learning?

The answers to the above questions seem to show no assessment of experiential learning at Namibian institutions of higher learning. What is currently being assessed through the MAE admission test is pure academic Mode 1 knowledge. It seems that assessors perceive that what they assess as inclusive of experiential learning in fact is not. Institutions need to decide whether or not to assess experiential learning and to adhere to their decisions in all aspects of requirements. The assessment of experiential learning is feasible and viable for entry purposes to institutions of higher learning. Procedures for admitting candidates to higher education through RPL are considered appropriate and beneficial. Assessors need to be trained in the existence of experiential learning and its assessment methods. Further studies should explore the feasibility of aligning experiential to level descriptors of the required NQF levels. Different knowledge forms do not only accommodate inclusivity but also facilitate wider access to higher education.

\section{References}

Breier, M. (2005). A Disciplinary-Specific Approach to the Recognition of Prior informal Experiences in Adult Pedagogy: 'rpl' as opposed to 'RPL'. Studies in Continuing Education, 27(1), 51-65.

Cohen, L., Manion, L., \& Morrison, K. (2010). Research Methods in Education. (6th ed.). New York, NY: Routledge.

Coles, A., \& McGrath, J. (2010). Your Education Research Project Handbook: ABC. Essex: Longman.

Conrad, D. (2010). Achieving Flexible learning through RPL practice: A Case study-lament of the Canadian academy. The Journal of Open Learning, 25(2), 153-161.

Creswell, J. W. (2009). Research Design: Qualitative, Quantitative, and Mixed Methods Approaches. (3rd ed.). Nebraska, NE: University of Nebraska-Lincoln.

Harris, J. (2000). RPL: Power Pedagogy and Possibility: Conceptual and implementation guide. Pretoria: Human Sciences Research Council. 
Harris, J. (1999). Ways of Seeing the Recognition of Prior Learning (RPL). What contribution can such practices make to social inclusion? Studies in the Education of Adults, 31(2), 124-142.

Henning, E., Smit S., \& Van Rensburg, W. (2004). Finding your way in academic research. (2nd ed.). Pretoria: Van Schaik.

Joosten-ten Brinke, D., Sluijsmans, D.M.A., \& Jochems, W.M.G. (2009a). Quality of Assessment of Prior Learning (RPL) in University Programmes. Perceptions of Candidates, Tutors and Assessors. Studies in Continuing Education, 31(1), 61-76.

Joosten-ten Brinke, D., Sluijsmans, D.M.A., \& Jochems, W.M.G. (2009b). Self- Assessment in University Assessment of Prior Learning procedures. Studies in Continuing Education, 28(1), 107-122.

Mankin, D. (2009). Human Resource Development. Oxford: Oxford University Press.

Merriam, S.B. (2009) Qualitative research. A guide to Design and Implementation. (Revised and expanded from Qualitative Research and Case study applications in Education). San Francisco, CA: Jossey- Bass.

Michelson, E., Mandell, A., \& Contributors. (2004). Portfolio Development and the Assessment of Prior Learning: Perspective, Model and Practice. (2 $2^{\text {nd }}$ ed.). Sterling, VA: Stylus Publish CLC.

Namibia. Ministry of Education and Culture. (1993). Towards Education for All: A Development Brief for Education Culture and Training. Windhoek: Gamsberg-Macmillan.

Namibia. Ministry of Education and Culture. (2006). Education Training Sector Improvement Programme (ETSIP). Windhoek: Gamsberg-Macmillan.

Namibia. Vision 2030. (2004) Policy Framework for Long-term National Development. Main Document. Windhoek: Office of the President.

Namibia Qualifications Authority (NQA). (2010). National Namibian RPL Policy. Windhoek: NQA.

National Council for Higher Education (NCHE). (2010). In Pursuit of Access with Equity in the Higher Education System of Namibia. A report of a Study conducted by NCHE. Windhoek: NCHE.

National Planning Commission of Namibia. (2008). Understanding the Third National Development Plan (NDP3). 2007/2008-2011/2012. Windhoek: Office of the President.

Neumann, W.L. (2011). Social Research Methods. Qualitative and Quantitative Approaches (7th ed.). Boston, MA: University of Wisconsin.

Ord, J. (2009). Experiential Learning in Youth Work in the UK: a return to Dewy. International Journal of Lifelong Learning, 28(4), 493-511.

Patton, M.Q. (2002). Qualitative Research and Evaluation Methods. s.1.: SAGE.

Salling-Olesen. H. (2007). Why is certification and validation important for lifelong learning? (An extract from a World Bank report: Certification and validation of Prior Learning and Competences with assistance from Torti, World Bank project. A paper presented at the world conference on LLL in Changwon city, Korea).

Stenlund, T. (2010). Assessment of Prior Learning in Higher Education: A Review from validity perspective. Assessment \& Evaluation in Higher Education, 35(7), 783-797.

Stuckey, L.H., Taylor, W. E., \& Cranton, P. (2014). Developing a Survey of Transformative Learning Outcomes and Processes Based on Theoretical Principles. Journal of Transformative Education, (11), 211.

Suopis, C.A. (2009). Changing PLA Processes, not PLA. The Journal of Containing Higher Education, 57(1), $57-128$.

University of Namibia (UNAM) (2012). Faculty of Education Prospectus 2012. Windhoek: UNAM, Inspiring Minds \& Shaping the Future.

Volbrecht, T. (2009). New Course for Trojan Horses: Re-Think RPL in a South African Teachers Education Curriculum: Cape Peninsula University of Technology South Africa. Studies in Community Education, 31(1), 13-27. 